Research Article

\title{
Quality Evaluation of Artemisia capillaris Thunb. Based on Qualitative Analysis of the HPLC Fingerprint and UFLC-Q-TOF-MS/MS Combined with Quantitative Analysis of Multicomponents
}

\author{
Ying Dai $\mathbb{D}^{1},{ }^{1}$ Zhihua Dou $\mathbb{D}^{1,2,3}$ Rongrong Zhou $\mathbb{D}^{1,},{ }^{2}$ Lin Luo, ${ }^{3}$ Li Bian, ${ }^{2}$ Yufeng Chen, \\ Jinhua Tao, ${ }^{3}$ and Zhixian Chen $\mathbb{D}^{4}$ \\ ${ }^{1}$ School of Pharmacy, Nanjing University of Chinese Medicine, Nanjing 210023, Jiangsu, China \\ ${ }^{2}$ Nantong Third People's Hospital, Nantong University, Nantong 226006, Jiangsu, China \\ ${ }^{3}$ School of Pharmacy, Nantong University, Nantong 226019, Jiangsu, China \\ ${ }^{4}$ Department of Pharmacy, Nantong Health College of Jiangsu Province, Nantong 226010, Jiangsu, China
}

Correspondence should be addressed to Rongrong Zhou; zhourongrong824@163.com and Zhixian Chen; zxchen81@163.com

Received 25 January 2021; Accepted 17 March 2021; Published 21 April 2021

Academic Editor: Eliseo Herrero Hernandez

Copyright $\left({ }_{0} 2021\right.$ Ying Dai et al. This is an open access article distributed under the Creative Commons Attribution License, which permits unrestricted use, distribution, and reproduction in any medium, provided the original work is properly cited.

In this study, a new method was developed for the comprehensive quality evaluation (QE) of Artemisia capillaris Thunb. (A. capillaris, named Yinchenhao in Chinese), which is one of the most commonly used herbal medicines (HMs). First, fingerprints of 31 batch samples of $A$. capillaris were determined by HPLC, the reference fingerprint was established, and the common peaks were assigned. Second, the components of common peaks in the HPLC fingerprints were identified by ultrafast liquid chromatography- (UFLC-) Q-TOF-MS/MS. Finally, the contents of the components unambiguously confirmed by reference substances were determined, and the correlation between the contents of chlorogenic acid and the contents of others was analyzed. The results showed that there were 20 common peaks in the HPLC fingerprints of 31 batch samples. The components of these 20 common peaks were identified as ten organic acids, eight flavonoids, and two others. Among nine organic acids such as 1-caffeoylquinic acid, neochlorogenic acid, chlorogenic acid, caffeic acid, cryptochlorogenic acid, 1,3-dicaffeoylquinic acid, 3,4-dicaffeoylquinic acid, 3,5-dicaffeoylquinic acid, and 4,5-dicaffeoylquinic acid, three flavonoids such as rutin, hyperoside, and isoquercetin, and one other $p$-hydroxyacetophenone, a total of 13 ones were unambiguously identified by comparison with reference substances; one caffeoylquinic acid glucoside and one flavone di-C-glucoside were detected in A. capillaris for the first time. There were some differences in the contents of 13 components in different samples; chlorogenic acid could be regarded as the quality marker of $A$. capillaris. The current established method in this study can be used for the comprehensive QE of A. capillaris and can also provide reference for the QE of the other HMs.

\section{Introduction}

The quality and quality evaluation (QE) method is crucial in the effectiveness and safety assessment of herbal medicines (HMs) [1]. The method for the QE of TCMs must be based on the holistic principle, and fingerprint describes integral characterization and reflects interactive aspects of complex components; therefore, it can offer the possibility of evaluating quality of HMs following the overall principle [2]. HPLC fingerprint has become the most widely used method due to its high reproducibility and sensitivity [3]. The HPLC fingerprint method applied to QE of HMs is mainly based on the similarity of fingerprints and the presence or absence of chromatographic peaks among samples $[4,5]$; however, this method cannot identify what these peaks are. Q-TOF-MS/ MS is a kind of tandem mass spectrometry providing a high mass resolution and accurate mass measurement for the structural elucidation of unknown chemicals [6] and can be used in the identification of common peaks in HPLC fingerprints. Based on the qualitative analysis of fingerprint, the 
quantitative analysis of multiple components is the key step of QE of HMs [5].

Artemisia capillaris Thunb. (A. capillaris, named Yinchenhao in Chinese) is one of the most commonly used HMs [7], which has been used in China, Korea, and Japan for a long time to treat liver and choleretic disorders, such as cholestasis, jaundice, liver fibrosis, and hepatitis [7-9]. The major components contained in A. capillaris include organic acids, flavonoids, coumarins, essential oil, and others, such as $p$-hydroxyacetophenone [7]. A characteristic fingerprint was developed to determine the volatile constituents in essential oil of $A$. capillaris by GC-MS [10], but the systematic study on the HPLC fingerprint of A. capillaris and the identification of common peaks by Q-TOF-MS have not been reported so far. In recent years, $\mathrm{QE}$ of A. capillaris based on multicomponents quantitative analysis has made some progress. Yu et al. developed a method to determine eight organic acids in A. capillaris extract by HPLC, including chlorogenic acid (CA), neochlorogenic acid (NCA), cryptochlorogenic acid (CCA), 1,3-dicaffeoylquinic acid (1,3-diCQA), 3,4-dicaffeoylquinic acid (3,4-diCQA), 3,5-dicaffeoylquinic acid (3,5diCQA), 4,5-dicaffeoylquinic acid (4,5-diCQA), and caffeic acid [11]. Tian et al. established a quantitative analysis method of six organic acids of NCA, CA, CCA, 1,3-diCQA, 3,4-diCQA, and 4,5-diCQA in A. capillaris and its decoction by HPLC [12]. Thirteen components including four organic acids, four flavonoids, four coumarins, and one other of $p$-hydroxyacetophenone and ten components including four organic acids, five flavonoids, and one coumarin of scoparone in A. capillaris were determined by the same method, respectively $[13,14]$. However, a comprehensive QE method for A. capillaris has not been established so far. Therefore, the aim of this work was to establish a new method for the $\mathrm{QE}$ of A. capillaris comprehensively based on qualitative analysis of the HPLC fingerprint and ultrafast liquid chromatography- (UFLC-) Q-TOF-MS/MS combined with quantitative analysis of multicomponents.

\section{Experimental}

2.1. Chemicals and Reagents. Reference substance of $p$ hydroxyacetophenone (no. 111897-201602, with purity $\geq 99.9 \%$ ) was purchased from the National Institutes for Food and Drug Control (Beijing, China). 1-caffeoylquinic acid (1-CQA, no. CHB170525), NCA (no. CHB170914), CA (no. CHB170713), caffeic acid (no. CHB160907), CCA (no. CHB170828), 1,3-diCQA (no. CHB160620), rutin (no. CHB170303), hyperoside (no. CHB160904), isoquercetin (no. CHB160912), 3,4-diCQA (no. CHB160725), 3,5-diCQA (no. CHB171013), and 4,5-diCQA (No. CHB160726) were purchased from the Chengdu Chroma Biotechnology Co., Ltd. (Chengdu, China) (all with purity $\geq 98 \%$ ). Methanol (HPLC grade) and acetonitrile (LC/MS grade) were purchased from Fisher Scientific (Fair Lawn, NJ, USA). Purified water was purchased from Wahaha
Group Co., Ltd. (Hangzhou, China). Formic acid (HPLC grade) was supplied by Nanjing Chemical Reagent Co. Ltd. (Nanjing, China).

2.2. Apparatus. Determination of the HPLC fingerprint and the contents of 13 components were performed on a HPLC system (Waters Corp., Milford, MA, USA), equipped with a Waters e2695 separation unit, a Waters 2998 PDA detector, and an Empower 3 data processing system. Chromatographic separation was performed on a Symmetry $\mathrm{C}_{18}$ column $(4.6 \mathrm{~mm} \times 250 \mathrm{~mm}, 5 \mu \mathrm{m}$, Waters Corp., USA). Acetonitrile $(A)$ and $0.1 \%(v / v)$ formic acid (B) were used as mobile phases with the following gradient elution: $0-35 \mathrm{~min}$, 5-10\% A; 35-65 min, 10-25\% A; 65-67 min, 25-90\% A; and $67-80 \mathrm{~min}, 90 \% \mathrm{~A}$. The flow rate was set at $1.0 \mathrm{~mL} / \mathrm{min}$, and the column temperature was maintained at $30^{\circ} \mathrm{C}$. The injection volume of sample solution was $30 \mu \mathrm{L}$. The detection wavelength of fingerprint and content of $p$-hydroxyacetophenone, rutin, hyperoside, and isoquercetin was set at $254 \mathrm{~nm}$ and that of content of nine organic acids was set at $324 \mathrm{~nm}$.

Identification of the common peaks in the HPLC fingerprint was performed on a UFLC-Q-TOF-MS/MS system. Separation was performed on a UFLC system (Shimadzu, Kyoto, Japan) by using a Symmetry $\mathrm{C}_{18}$ column $(250 \mathrm{~mm} \times 4.6 \mathrm{~mm}, 5 \mu \mathrm{m})$; with the same mobile phases and the same gradient conditions abovementioned, the injection volume of the mixed reference substances solution for qualitative analysis and sample solution was all $20 \mu \mathrm{L}$. After separation, mass spectra were acquired on the AB Triple TOF 4600 plus system (AB SCIEX, Framingham, USA) with the following mass spectrometric parameters: ion source, DuoSpray; ESI mode, negative; ion source temperature, $550^{\circ} \mathrm{C}$; ion spray voltage, $-4500 \mathrm{~V}$; nebulizer gas (gas 1 ), 60 psi; heater gas (gas 2), 60 psi; and curtain gas (CUR), 35 psi. The TOFMS-IDA-10MS/MS information acquisition method was used to obtain mass spectrometry information, and the parameters were set as follows: decluster potential (DP) of $-80 \mathrm{~V}$, collision energy (CE) of $-10 \mathrm{eV}$, accumulation time of $250 \mathrm{~ms}$, mass range of $105-1500 \mathrm{Da}$ for the TOF-MS scan, collision energy (CE) of $-35 \mathrm{eV}$, collision energy spread (CES) of $15 \mathrm{eV}$, and mass range of 50-1500 Da for the TOF-MS/MS detection. LC-MS/MS data were analyzed using PeakView mass spectrometry analysis software (Version 1.6, AB SCIEX, USA).

2.3. Samples and Sample Preparation. Information on all 31 batches of samples is given in Table 1, among which, 30 batches of $A$. capillaris (S1-S30) were purchased from different large TCM hospitals in China and authenticated as the dried aerial part of $A$. capillaris by the chief Chinese pharmacist Xudong Gong, the director of the Nantong Food and Drug Supervision and Inspection Centre. Herbal reference substance of $A$. capillaris (S31) was purchased from the National Institutes for Food and Drug Control (Beijing, China) in 2018. 
Table 1: Samples information and similarities.

\begin{tabular}{|c|c|c|c|c|}
\hline Sample no. & Manufacturers & Batch no. & Origins of herb & Similarity \\
\hline S1 & Nantong Sanyue Herbal Pieces Co., Ltd. & 171208 & Henan & 0.949 \\
\hline S2 & Nantong Sanyue Herbal Pieces Co., Ltd. & 180109 & Henan & 0.993 \\
\hline S3 & Nantong Sanyue Herbal Pieces Co., Ltd. & 151110 & Henan & 0.996 \\
\hline S4 & Nantong Sanyue Herbal Pieces Co., Ltd. & 180308 & Henan & 0.905 \\
\hline S5 & Suzhou Tianling Herbal Pieces Co., Ltd. & 180205 & Jiangsu & 0.958 \\
\hline S6 & Suzhou Tianling Herbal Pieces Co., Ltd. & 180205010 & Jiangsu & 0.963 \\
\hline S7 & Suzhou Tianling Herbal Pieces Co., Ltd. & 170902010 & Jiangsu & 0.980 \\
\hline S8 & Suzhou Tianling Herbal Pieces Co., Ltd. & 180205015 & Jiangsu & 0.969 \\
\hline S9 & Suzhou Tianling Herbal Pieces Co., Ltd. & 160309010 & Jiangsu & 0.963 \\
\hline S10 & Suzhou Tianling Herbal Pieces Co., Ltd. & 160122010 & Jiangsu & 0.990 \\
\hline S11 & Yancheng Herbal Pieces Co., Ltd. & 2017091402 & Jiangsu & 0.986 \\
\hline S12 & Anhui Bozhou Qiancao Guoyao Co., Ltd. & 1712176 & Shanxi & 0.991 \\
\hline S13 & Anhui Wansheng Herbal Pieces Co., Ltd. & 171201 & Shanxi & 0.994 \\
\hline S14 & Anhui Wansheng Herbal Pieces Co., Ltd. & 180302 & Shanxi & 0.928 \\
\hline S15 & Anhui Mengshi Herbal Pieces Co., Ltd. & 170401 & Anhui & 0.961 \\
\hline S16 & Bozhou Qiaocheng Wanshixiang Herbal Pieces Co., Ltd. & 170901 & Shanxi & 0.970 \\
\hline S17 & Anhui Xiehecheng Pharmaceutical Herbal Pieces Co., Ltd. & 17051814 & Shanxi & 0.986 \\
\hline S18 & Anhui Shenghaitang Herbal Pieces Co., Ltd. & 2017080421 & Shanxi & 0.991 \\
\hline S19 & Hebei Kaida Pharmaceutical Co., Ltd. & 20171201 & Hebei & 0.987 \\
\hline S20 & Zhejiang Tongjuntang Herbal Pieces Co., Ltd. & 170921 & Shaanxi & 0.996 \\
\hline S21 & Zhengzhou Ruilong Pharmaceutical Co., Ltd. & 17110102 & Henan & 0.990 \\
\hline S22 & Hebei Renxin Pharmaceutical Co., Ltd. & 21118001 & Hebei & 0.983 \\
\hline S23 & Shaohuatang Guoyao Co., Ltd. & 171010 & Shanxi & 0.975 \\
\hline S24 & Shanghai Wanshicheng Guoyao Products Co., Ltd. & $180418-1$ & Shandong & 0.934 \\
\hline S25 & Shanghai Kangqiao Herbal Pieces Co., Ltd. & 171125 & Shaanxi & 0.972 \\
\hline S26 & Yancheng Herbal Pieces Co., Ltd. & 2016030202 & Jiangsu & 0.994 \\
\hline S27 & Hangzhou Huadong Herbal Pieces Co., Ltd. & 1601118 & Shandong & 0.953 \\
\hline S28 & Bozhou Yonggang Herbal Pieces Co., Ltd. & 15113001 & Shanxi & 0.991 \\
\hline S29 & Bozhou Yonggang Herbal Pieces Co., Ltd. & 15070901 & Shanxi & 0.994 \\
\hline S30 & Yunnan Baiyao Herbal Medicine Branch of Yunnan Group Co., Ltd. & YP20140601 & Hubei & 0.983 \\
\hline S31 & National Institutes for Food and Drug Control & $121555-201101$ & Unknown & 0.968 \\
\hline
\end{tabular}

The samples were dried at $40^{\circ} \mathrm{C}$, ground into powder, and then sieved through a 40-mesh screen. Approximately $0.2 \mathrm{~g}$ of sample powder was accurately weighed and placed in a $50 \mathrm{~mL}$ dark brown volumetric flask. Approximately $49 \mathrm{~mL}$ of $50 \%(v / v)$ methanol was added and extracted by ultrasonication $(200 \mathrm{~W}, 53 \mathrm{kHz})$ for $30 \mathrm{~min}$. After cooling to room temperature, $50 \%(v / v)$ methanol was added for calibration of the volumetric flask and shaken well. The extract was filtered through a $0.22 \mu \mathrm{m}$ filter membrane, and the filtrate was taken as the sample solution.

2.4. Preparation of Reference Substance Solutions. Appropriate amounts (5-20 mg) of 13 reference substances were accurately weighted, dissolved with 50\% ( $v / v)$ methanol, respectively, and 13 reference substance stock solutions were prepared.

The mixed reference substances solution for qualitative analysis with a concentration range of $0.4-50 \mu \mathrm{g} / \mathrm{mL}$ of each compound was prepared by accurately absorbing appropriate volume of 13 reference substance stock solutions, mixing them, and diluting the mixture with $50 \%(v / v)$ methanol.

Working solution A for quantitative analysis was prepared by the same method as the mixed reference substances solution for qualitative analysis, and the final concentrations of 13 reference substances were at the range of 3.1-193 $\mu \mathrm{g} /$ $\mathrm{mL}$. Working solution A was diluted two, five, and ten times with $50 \%(v / v)$ methanol to prepare working solutions $\mathrm{B}, \mathrm{C}$, and $\mathrm{D}$, respectively.

2.5. Method Validation of the HPLC Fingerprint Analysis. The method of HPLC fingerprint determination was validated with precision, stability, and repeatability tests, by using peak 5 (CA) as the reference peak and the relative standard deviation (RSD) value of the average relative retention time (RRT) and relative peak area (RPA) of the 20 common peaks as measure values. In the precision test, six consecutive injections of the same sample (S1) solution were analyzed. Stability was examined by analyzing the sample solution (S1) at $0,6,12,18,24$, and $36 \mathrm{~h}$ after preparation. Repeatability was examined by determination of six sample solutions prepared in parallel from S1.

2.6. Method Validation of the Quantitative Analysis. The method of quantitative analysis was validated with investigation of linear relationships, limit of quantitation (LOQ), limit of detection (LOD), precision, stability, repeatability, and the recovery test of 13 components. Investigation of linear relationships was performed by precisely injecting working solutions $\mathrm{B}, \mathrm{C}$, and $\mathrm{D} 10 \mu \mathrm{L}$ 
TABLE 2: Results of the investigation of the linear relationship, LOD, and LOQ.

\begin{tabular}{|c|c|c|c|c|c|}
\hline Reference substance & Regression equation & $R^{2}$ & Linear range (ng) & LOD (ng) & LOQ (ng) \\
\hline 1-CQA & $Y=2231204 X-8098$ & 0.9999 & $13.35 \sim 534$ & 1.60 & 5.34 \\
\hline $\mathrm{NCA}$ & $Y=2830658 X-5170$ & 0.9999 & $5.15 \sim 206$ & 0.34 & 1.72 \\
\hline $\mathrm{CA}$ & $Y=2870573 X-117405$ & 0.9999 & $192.3 \sim 7692$ & 1.54 & 7.69 \\
\hline Caffeic acid & $Y=4958993 X-7629$ & 0.9998 & $1.90 \sim 76.05$ & 0.30 & 1.52 \\
\hline CCA & $Y=2656572 X-12630$ & 0.9998 & $7.66 \sim 306.24$ & 0.51 & 2.55 \\
\hline p-Hydroxyacetophenone & $Y=2885362 X-7875$ & 0.9999 & $3.05 \sim 121.92$ & 0.41 & 1.63 \\
\hline 1,3-DiCQA & $Y=2995412 X-1885$ & 0.9999 & $1.77 \sim 70.84$ & 0.24 & 1.18 \\
\hline Rutin & $Y=1638734 X-1384$ & 0.9999 & $2.53 \sim 101$ & 0.40 & 1.68 \\
\hline Hyperoside & $Y=2406676 X-5708$ & 0.9999 & $8.31 \sim 332.4$ & 0.33 & 1.66 \\
\hline Isoquercetin & $Y=1973858 X-1855$ & 0.9999 & $2.90 \sim 116$ & 0.23 & 1.74 \\
\hline 3,4-DiCQA & $Y=2669900 X-7344$ & 0.9999 & $5.04 \sim 201.6$ & 0.67 & 2.69 \\
\hline 3,5-DiCQA & $Y=3267110 X+5860$ & 0.9999 & $122.30 \sim 4892$ & 1.73 & 3.46 \\
\hline 4,5-DiCQA & $Y=2863719 X-7095$ & 0.9999 & $13.65 \sim 546$ & 1.09 & 5.46 \\
\hline
\end{tabular}

and working solution A 10, 20, 30, and $40 \mu \mathrm{L}$ into the HPLC systems for the calculation of the regression equations, correlation coefficients, and linear ranges of 13 components. Working solution D was successively diluted with $50 \%(v / v)$ methanol to give different concentrations of reference substance solutions. The LOQ and LOD values were determined by using signal-to-noise ratios of $3: 1$ and $10: 1$ and injecting $10 \mu \mathrm{L}$ of above different concentrations of reference substance solutions. By using the RSDs of the peak areas of the 13 components as the measurement values, intraday precision, interday precision, and stability tests were performed, respectively. In the intraday precision test, six consecutive injections of $30 \mu \mathrm{L}$ working solution A were analyzed, and in the interday precision test, six injections of $30 \mu \mathrm{L}$ working solution A were analyzed twice a day over three consecutive days. Stability was examined by analyzing the peak areas of nine organic acids at $324 \mathrm{~nm}$ and four others at $254 \mathrm{~nm}$ detected in Section 2.5 of the stability test. Repeatability was examined by calculating the contents of 13 components according to the peak areas of nine organic acids at $324 \mathrm{~nm}$ and four others at $254 \mathrm{~nm}$ detected in Section 2.5 of the repeatability test and using the RSDs of the contents as the measured values. In the recovery test, approximately $0.1 \mathrm{~g}$ of S1 powder was weighed precisely, and then, 13 reference substance stock solutions were added to the sample in a certain volume according to the approximate proportion of the sample content to the reference substance $(1: 1)$ to prepare six sample solutions in parallel. The six sample solutions were injected into HPLC, and the average recovery rates and RSDs of the 13 components were calculated.

We followed the methods of Author links open overlay panel [15].

\section{Results and Discussion}

3.1. Method Validation of the HPLC Fingerprint Analysis. The RSDs of RRT and RPA for precision were less than $0.10 \%$ and $4.4 \%$, those of stability were no more than $0.08 \%$ and $4.7 \%$, and those of repeatability did not exceed $0.08 \%$ and $4.8 \%$, respectively. The results met the national standards of TCM fingerprinting [16].
3.2. Method Validation of the Quantitative Analysis. As given in Table 2, the high correlation coefficient values $\left(R^{2}>\right.$ 0.9998 ) displayed good linearity over a wide range of injected amounts, and as given in Table 3, the RSDs of the intraday precision, interday precision, stability, and repeatability were all less than $5 \%$, and the average recovery rates were in the range of $95.49 \%-103.20 \%$ with RSD values ranging $0.90-4.95 \%$. The above results met the requirements of drug quality standard analysis method in Chinese Pharmacopoeia [17].

3.3. Establishment of the HPLC Fingerprint and Similarity Analysis. 31 batches of $A$. capillaris samples were determined (chromatograms are shown in Figure 1). The chromatographic data of the samples were imported into the software of Similarity Evaluation System for Chromatographic Fingerprint of Traditional Chinese Medicine (version 2012, Chinese Pharmacopoeia Commission, Beijing, China). Using the chromatogram of $\mathrm{S} 1$ as a reference, the reference chromatogram was generated, and 20 peaks were extracted to be the common peaks (shown in Figure 1). The similarities between sample chromatograms and reference chromatogram were calculated by the above software, and the results showed that the similarities of 31 batches of A. capillaris were all greater than 0.9 (Table 3).

\subsection{Identification of the Common Peaks by UFLC-Q-TOF-MS/} MS. Since more information and higher identifiability of fragmentation was observed in the negative ion mode, it was chosen for MS analysis rather than the positive ion mode. First, total ion chromatograms of the A. capillaris sample and mixed reference substances (Figure 2) were extracted using PeakView mass spectrometry analysis software. Second, the mass spectral data and dissociative rules of the reference substances were summarized, and the law of the quasimolecular ion $[\mathrm{M}-\mathrm{H}]^{-}$and/or $[\mathrm{M}+\mathrm{Cl}]^{-}$that could be selected as the precursor ion for collision-induced dissociation fragmentation to produce MS/MS product ions spectra was revealed. Finally, the components of the total 20 common peaks in the HPLC fingerprint were identified by comparing the retention time, $m / z$ of $[\mathrm{M}-\mathrm{H}]^{-}$and/or 
TABLE 3: Results of precision, stability, repeatability, and recovery tests $(n=6)$.

\begin{tabular}{|c|c|c|c|c|c|c|}
\hline \multirow{2}{*}{ Components } & \multicolumn{2}{|c|}{ Precision RSD (\%) } & \multirow{2}{*}{ Stability RSD (\%) } & \multirow{2}{*}{ Repeatability (RSD, \%) } & \multicolumn{2}{|c|}{ Recovery } \\
\hline & Intraday & Interday & & & Mean (\%) & RSD (\%) \\
\hline 1-CQA & 0.51 & 0.37 & 0.71 & 2.16 & 101.23 & 2.11 \\
\hline $\mathrm{NCA}$ & 0.66 & 0.69 & 2.26 & 3.82 & 97.68 & 0.90 \\
\hline $\mathrm{CA}$ & 0.53 & 0.52 & 0.26 & 4.17 & 96.19 & 3.09 \\
\hline Caffeic acid & 0.73 & 0.78 & 1.14 & 1.17 & 97.41 & 2.59 \\
\hline CCA & 0.58 & 0.49 & 1.03 & 3.93 & 99.66 & 4.89 \\
\hline$p$-Hydroxyacetophenone & 0.67 & 0.70 & 1.20 & 1.83 & 97.15 & 3.00 \\
\hline 1,3-DiCQA & 0.72 & 0.76 & 2.30 & 3.64 & 95.49 & 4.78 \\
\hline Rutin & 2.84 & 0.92 & 3.59 & 2.22 & 96.68 & 4.32 \\
\hline Hyperoside & 0.65 & 0.56 & 0.35 & 3.97 & 95.75 & 4.77 \\
\hline Isoquercetin & 0.86 & 0.78 & 2.85 & 4.06 & 98.47 & 4.95 \\
\hline 3,4-DiCQA & 0.62 & 0.62 & 4.69 & 1.16 & 103.20 & 4.85 \\
\hline 3,5-DiCQA & 0.62 & 0.65 & 0.71 & 4.02 & 98.96 & 3.75 \\
\hline 4,5-DiCQA & 0.60 & 0.61 & 0.53 & 3.81 & 96.83 & 4.82 \\
\hline
\end{tabular}

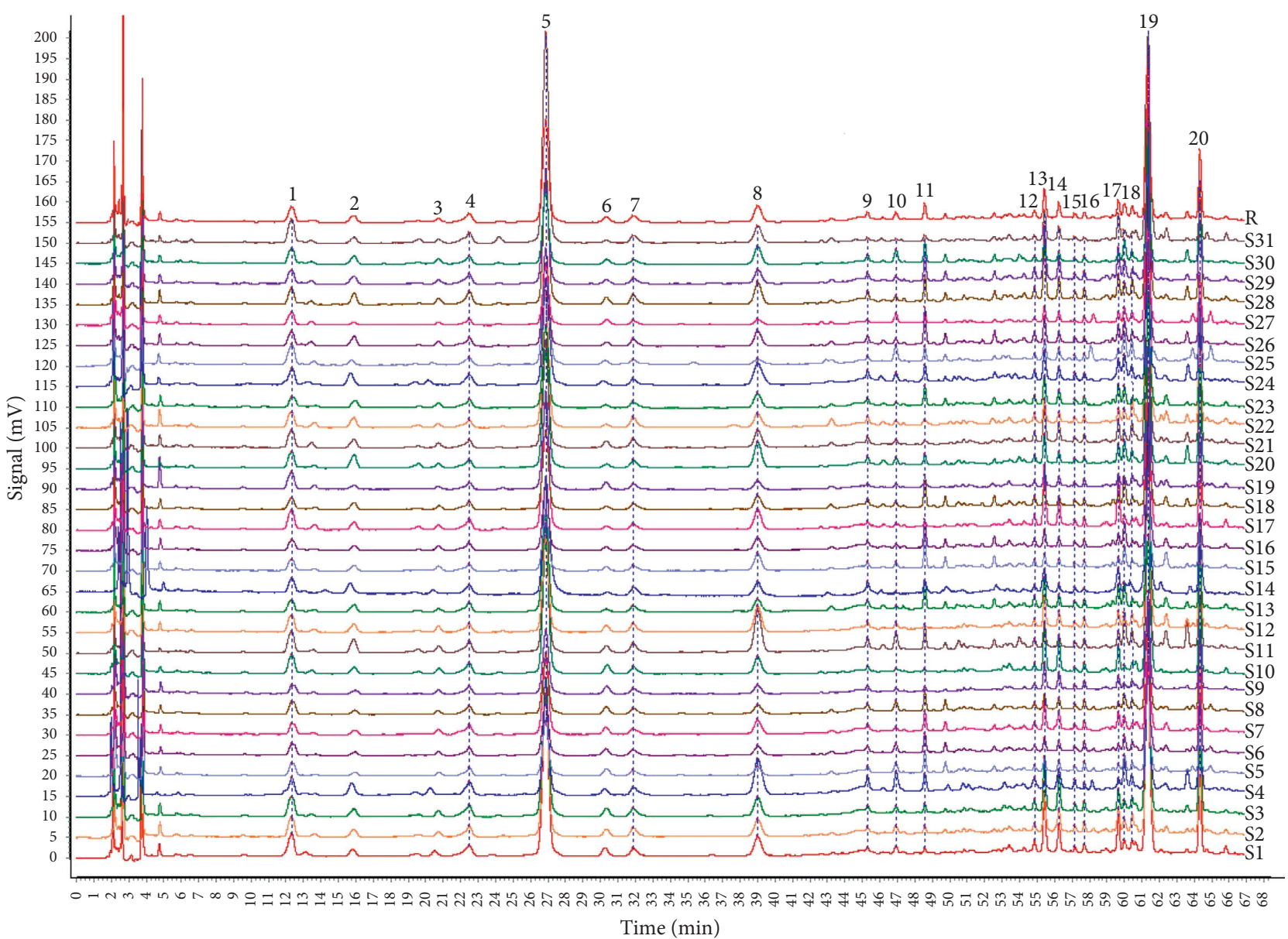

FIGURE 1: Chromatograms of 31 batches of A. capillaris (S1-S31) and the reference chromatogram (R).

$[\mathrm{M}+\mathrm{Cl}]^{-}$and $\mathrm{MS} / \mathrm{MS}$ fragmentation patterns with those of the reference substances or previous literature reports, combining with online retrieval of two compound database of PubChem (http://pubchem.ncbi.nlm.nih.gov) and ChemSpider (http://www.chemsipider. com). The mass spectral data are given in Table 4 .
Among 20 components, 13 ones were unambiguously identified by comparison with the reference substances, including nine organic acids such as 1-CQA (peak 1), NCA (peak 2), CA (peak 5), caffeic acid (peak 6), CCA (peak 7), 1,3-diCQA (peak 10), 3,4-diCQA (peak 18), 3,5-diCQA (peak 19), and 4,5-diCQA (peak 20), three flavonoids such as 


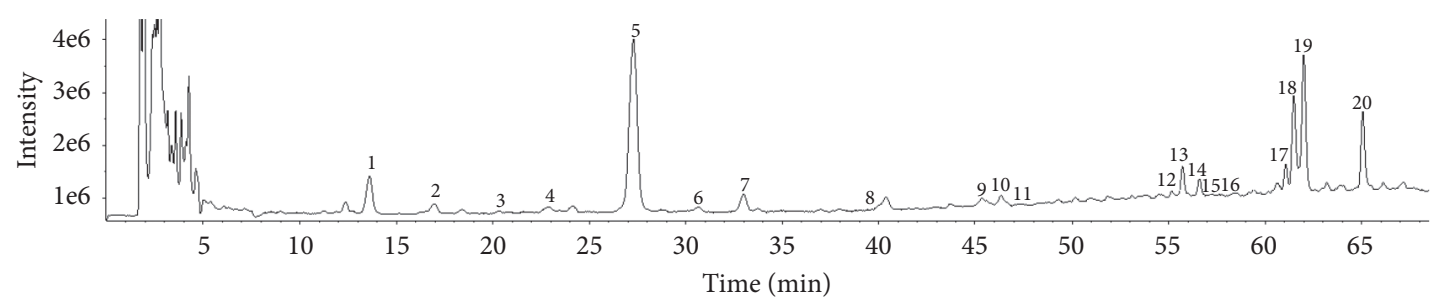

(a)

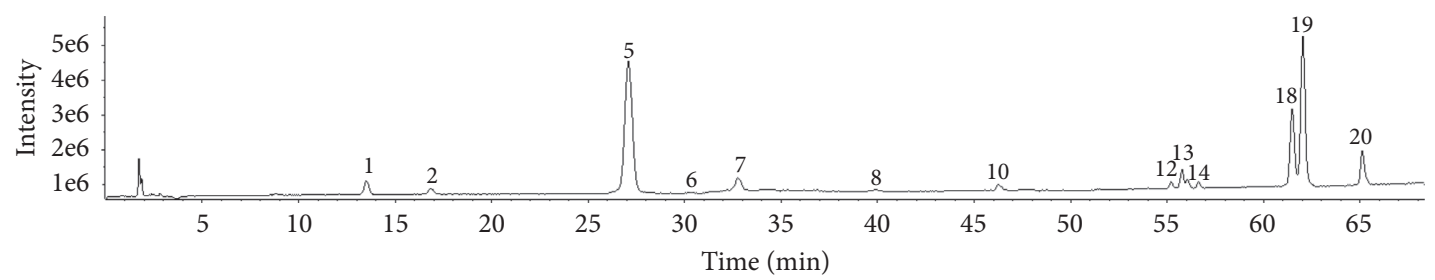

(b)

FIgURE 2: Total ion chromatogram of A. capillaris sample (a) and mixed reference substances (b) (negative ion mode).

TABLe 4: Identification of the common peaks in the A. capillaris fingerprint by UHLC-Q-TOF-MS/MS.

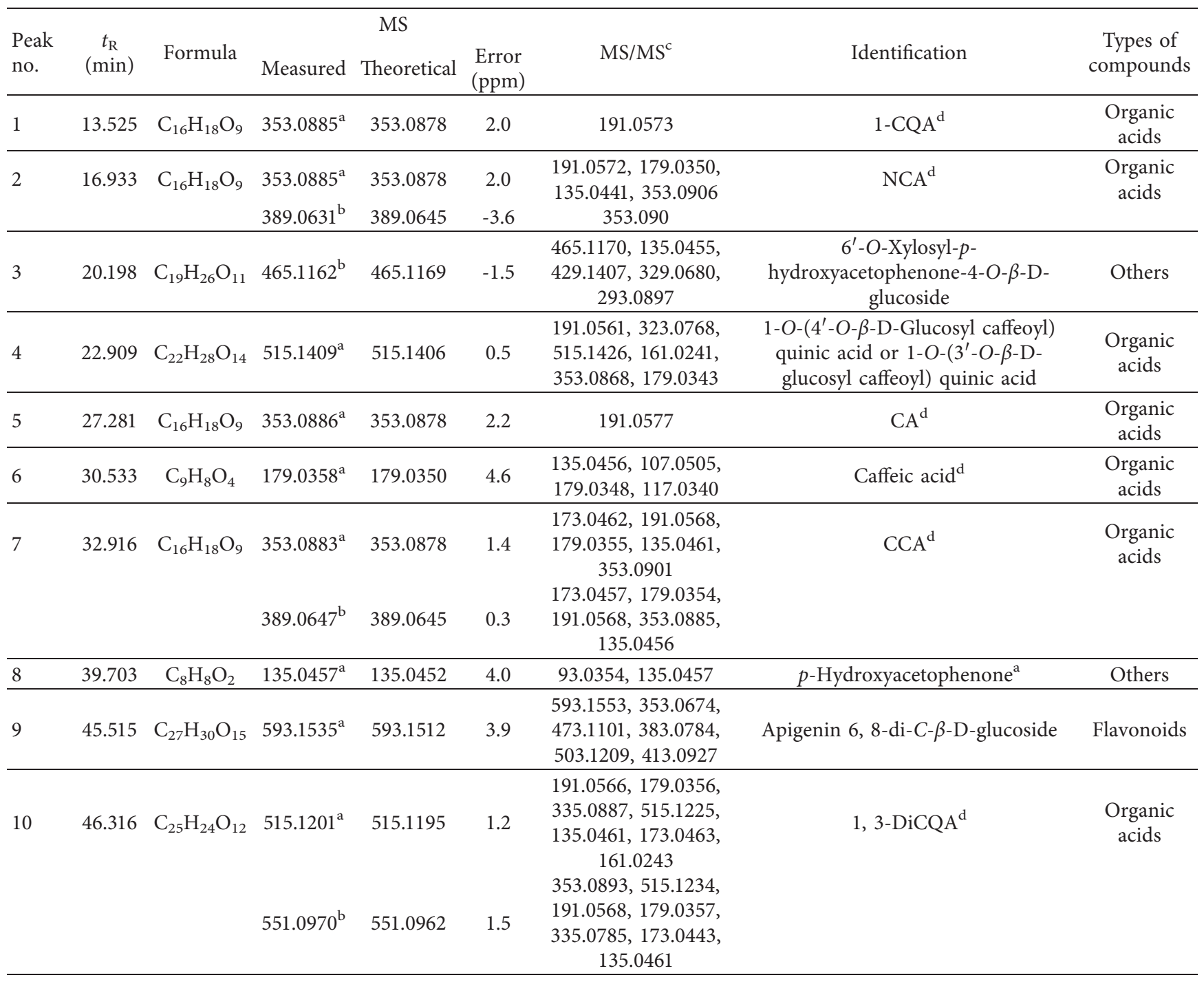


TABLE 4: Continued.

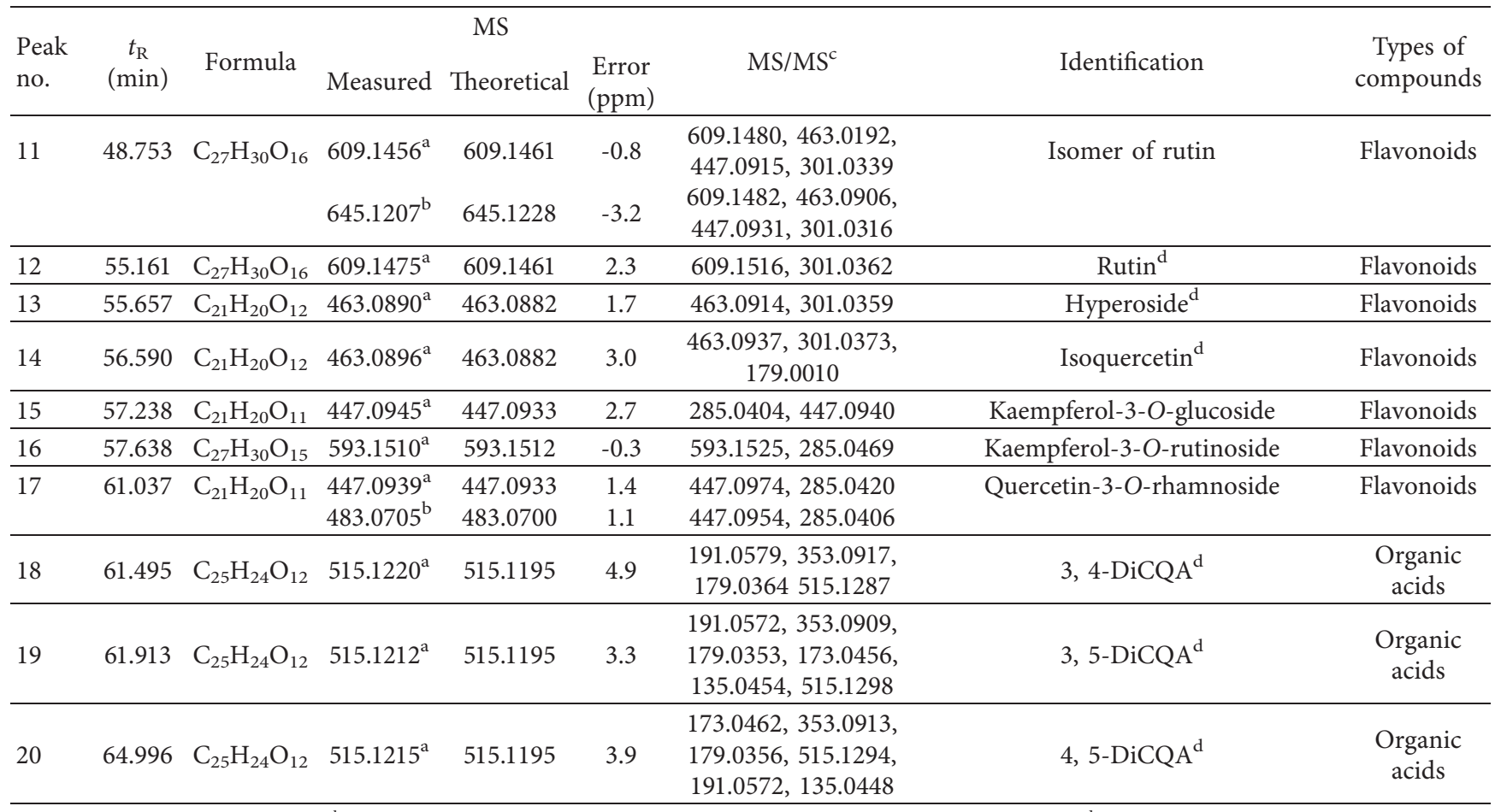

Quasimolecular ion was $[\mathrm{M}-\mathrm{H}]^{-}$. ${ }^{\mathrm{b}}$ Quasimolecular ion was $[\mathrm{M}+\mathrm{Cl}]^{-}$. ${ }^{\mathrm{c}}$ Sequencing according to the abundance. ${ }^{\mathrm{d}}$ Confirmed by comparison with reference substances.<smiles>O=c1cc(-c2ccc(O)cc2)oc2c(C3O[C@H](CO)[C@@H](O)[C@H](O)[C@H]3O)c(O)c(CC(O)=CO)c(O)c12</smiles>

$m / z 503$<smiles>O=c1cc(-c2ccc(O)cc2)oc2c(CC(O)CO)c(O)c(C[C@H](O)CO)c(O)c12</smiles>

$m / z 413$<smiles>[3H]C([3H])(O)c1ccc(-c2cc(=O)c3c(O)c(C4O[C@H](CO)[C@@H](O)[C@H](O)[C@H]4O)c(O)c(C4O[C@H](CO)[C@@H](O)[C@H](O)[C@H]4O)c3o2)cc1</smiles>

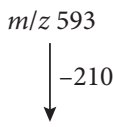<smiles>O=c1cc(-c2ccc(O)cc2)oc2c(CCO)c(O)c(/C=C\O)c(O)c12</smiles>

$m / z 383$<smiles>O=c1cc(-c2ccc(O)cc2)oc2c(C3O[C@H](CO)[C@@H](O)[C@H](O)[C@H]3O)c(O)c(/C=C\O)c(O)c12</smiles>

$m / z 473$

Figure 3: The possible dissociation pathway of the component of peak 9.

rutin (peak 12), hyperoside (peak 13), and isoquercetin (peak 14), and one other $p$-hydroxyacetophenone (peak 8).

For peak 3 , the molecular formula of $\mathrm{C}_{19} \mathrm{H}_{26} \mathrm{O}_{11}$ was speculated by software, and its quasimolecular ion was at an $\mathrm{m} /$ $z$ of $465.1162\left([\mathrm{M}+\mathrm{Cl}]^{-}\right)$. No literature reported the compounds with the molecular formula of $\mathrm{C}_{19} \mathrm{H}_{26} \mathrm{O}_{11}$ in A. capillaris so far. Sixty-nine and twenty-seven compounds consistent with this formula were retrieved from PubChem and ChemSpider, respectively. The structures of these compounds were analyzed one by one by the exclusion method, combined with $p$-hydroxyacetophenone and $6^{\prime}$ - $O$-dicaffeoyl- $p$ - hydroxyacetophenone-4-O- $\beta$-D-glucoside, and another compound with same parent nucleus [18], existed in A. capillaris; peak 3 was temporarily identified as 4-acetylphenyl 6-O- $\beta$-D-xylosyl- 
<smiles>[R20]OC1CC(O[R20])(C(=O)O)CC([R20])C1O</smiles><smiles>CC(=O)/C=C/c1ccc(O)c(O)c1</smiles>

$\begin{array}{ccccc}\text { Peak no. } & \mathrm{R}_{1} & \mathrm{R}_{2} & \mathrm{R}_{3} & \mathrm{R}_{4} \\ 1 & \text { Caffeyl } & \mathrm{H} & \mathrm{H} & \mathrm{H} \\ 2 & \mathrm{H} & \mathrm{H} & \mathrm{H} & \text { Caffeyl } \\ 5 & \mathrm{H} & \text { Caffeyl } & \mathrm{H} & \mathrm{H} \\ 7 & \mathrm{H} & \mathrm{H} & \text { Caffeyl } & \mathrm{H} \\ 10 & \text { Caffey1 } & \text { Caffeyl } & \mathrm{H} & \mathrm{H} \\ 18 & \mathrm{H} & \text { Caffeyl } & \text { Caffeyl } & \mathrm{H} \\ 19 & \mathrm{H} & \text { Caffeyl } & \mathrm{H} & \text { Caffeyl } \\ 20 & \mathrm{H} & \mathrm{H} & \text { Caffeyl } & \text { Caffeyl }\end{array}$<smiles>CCOc1ccc(/C=C/C(=O)OC2(C(=O)O)CC(O)C(O)C(O)C2)cc1OC</smiles>

$\mathrm{R}_{1}=\mathrm{H}, \mathrm{R}_{2}=$ glucosyl or $\mathrm{R}_{1}=$ glucosy $1, \mathrm{R}_{2}=\mathrm{H}$ Peak 4<smiles>CC(=O)c1ccc(O)cc1</smiles>

Peak 8<smiles>CO[C@H]1O[C@H](CO)[C@@H](O)[C@H](O)[C@H]1O</smiles><smiles>O=C(O)/C=C/c1ccc(O)c(O)c1</smiles>

Peak 6

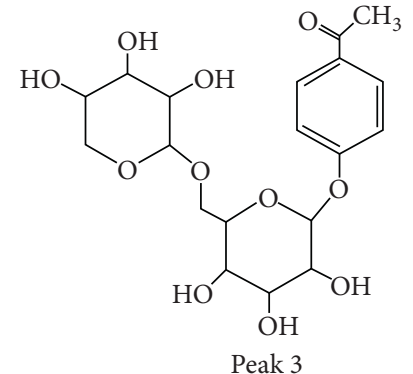<smiles>[R]c1cc(-c2oc3cc(O)cc(O)c3c(=O)c2[R])ccc1O</smiles>

$\begin{array}{ccc}\text { Peak no. } & \mathrm{R}_{1} & \mathrm{R}_{2} \\ 11 & \mathrm{OH} & \text { Rutinosesy1 } \\ 12 & \mathrm{OH} & \text { Rutinosesy1 } \\ 13 & \mathrm{OH} & \text { Galactosy1 } \\ 14 & \mathrm{OH} & \text { Glucosy1 } \\ 15 & \mathrm{H} & \text { Glucosy1 } \\ 16 & \mathrm{H} & \text { Rutinosesy1 } \\ 17 & \mathrm{OH} & \text { Rhamnosy1 }\end{array}$

FIGURE 4: Structures or possible structures of the components of peaks 1-8 and peaks 10-20.

$\beta$-D-glucoside (6'-O-xylosyl- $p$-hydroxyacetophenone-4-O$\beta$-D-glucoside), the first compound in both databases. In MS/MS spectrum of this compound, $\mathrm{m} / \mathrm{z}$ of 465.1170 , $429.1407,329.0680,293.0897$, and 135.0455 were determined, which was corresponded to $[\mathrm{M}+\mathrm{Cl}]^{-},[\mathrm{M}-\mathrm{H}]^{-}$, $[\mathrm{M}+\mathrm{Cl}]^{-}$loss of p-hydroxyacetophenone $\left(\mathrm{C}_{8} \mathrm{H}_{8} \mathrm{O}_{2}\right)$, $[\mathrm{M}-\mathrm{H}]^{-}$loss of $\mathrm{C}_{8} \mathrm{H}_{8} \mathrm{O}_{2}$, and $[\mathrm{M}-\mathrm{H}]^{-}$loss of xyloseglucosyl $\left(\mathrm{C}_{11} \mathrm{H}_{18} \mathrm{O}_{9}\right)$, respectively.

According to literature [19], the component of peak 4 was identified as a caffeoylquinic acid glucoside. The quasimolecular ion of this component was at an $\mathrm{m} / \mathrm{z}$ of $515.1409\left([\mathrm{M}-\mathrm{H}]^{-}\right)$, and the MS/MS fragment ions were determined as an $\mathrm{m} / z$ of 515.1426 corresponding to [M$\mathrm{H}]^{-}$, an $\mathrm{m} / z$ of 353.0868 corresponding to $\left[515-\mathrm{C}_{6} \mathrm{H}_{10} \mathrm{O}_{5}\right.$ (glucosyl)] $]^{-}$, an $\mathrm{m} / z$ of 323.0768 corresponding to [515$\mathrm{C}_{7} \mathrm{H}_{12} \mathrm{O}_{6}$ (quinic acid) $]^{-}$, an $\mathrm{m} / z$ of 191.0561 corresponding to [353- $\mathrm{C}_{9} \mathrm{H}_{6} \mathrm{O}_{3}$ (caffeyl) $]^{-}$, an $\mathrm{m} / z$ of 179.0343 corresponding to $\left[353-\mathrm{C}_{7} \mathrm{H}_{10} \mathrm{O}_{5} \text { (residue of quinic acid) }\right]^{-}$, and an $\mathrm{m} / z$ of $161.0241\left[353-\mathrm{C}_{7} \mathrm{H}_{12} \mathrm{O}_{6}\right]^{-}$. The linkage position between caffeoyl and quinic acid could be distinguished based on the $\mathrm{MS}^{2}$ fragmentation; when this position was at $1-\mathrm{OH}, 3-\mathrm{OH}$, or $5-\mathrm{OH}$, the $\mathrm{m} / z$ of 191 was the base peak; while linkage position was at 4-OH, the $\mathrm{m} / \mathrm{z}$ of 173 was the base peak [19]. An $m / z$ of 191 was determined as the base peak of peak 4 , so the connection position of $4-\mathrm{OH}$ was excluded. The relative intensity of $\mathrm{m} / z 179$ fragment ion could also be used to determine the linkage position between caffeoyl and quinic acid [19]. The relative intensity of $\mathrm{m} / z 179$ fragment ion of peak 4 was determined as $5.62 \%$, the one of 1-CQA (peak 1) and NCA (peak 2, linkage position was at 5-OH) was determined as $6.06 \%$ and $55.28 \%$, respectively, and fragment ion of $\mathrm{m} / \mathrm{z}$ 179 was not detected in CA (peak 5, linkage position was at $3-\mathrm{OH})$. Therefore, the component of peak 4 was temporarily identified as $1-O-\left(4^{\prime}-O-\beta-D\right.$-glucosyl caffeoyl $)$ quinic acid or $1-O-\left(3^{\prime}-O-\beta\right.$-D-glucosyl caffeoyl) quinic acid, which was first detected in A. capillaris, to the best of our knowledge. 


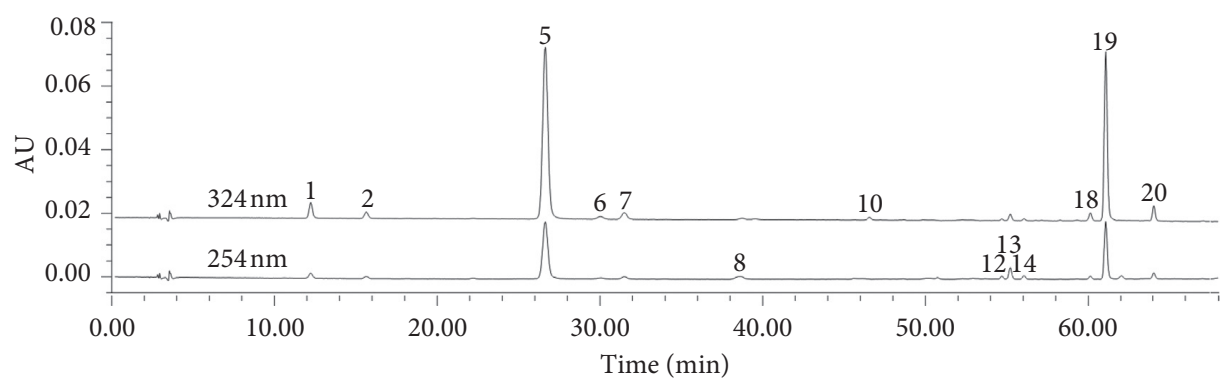

(a)

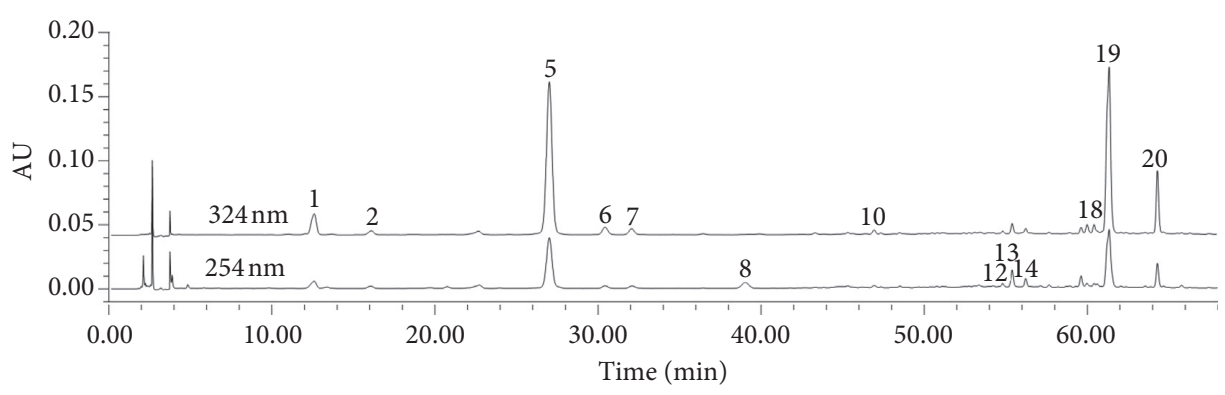

(b)

Figure 5: HPLC chromatograms of the mixed reference substances (a) and A. capillaris sample (b). The number of peaks is the same as Table 4.

The component of peak 9 was a typical flavone di-Cglucoside, according to the analysis of detected mass spectrometry data and literature [20]. The quasimolecular ion of this component was determined as an $\mathrm{m} / \mathrm{z}$ of 593.1535 $\left([\mathrm{M}-\mathrm{H}]^{-}\right)$, and the MS/MS fragment ions were $\mathrm{m} / z$ of 593.1553, 503.1209, 473.1101, 413.0927, 383.0784, and 353.0674 , which were consistent with the mass spectrum data of apigenin 6,8 -di- $C$ - $\beta$-D-glucoside reported in the literature [20], and its possible dissociation pathway is shown in Figure 3. To the best of our knowledge, this component was also first detected in A. capillaris.

The component of peak 11 was identified as the isomer of rutin according to literature [21]. Its quasimolecular ion was determined as an $\mathrm{m} / z$ of $609.1456\left([\mathrm{M}-\mathrm{H}]^{-}\right)$and an $\mathrm{m} / z$ of $645.1207\left([\mathrm{M}+\mathrm{Cl}]^{-}\right)$, and its MS/MS fragment ions were $\mathrm{m} / z$ of $609.1480,447.0915$, and 301.0339, which correspond to $[\mathrm{M}-\mathrm{H}]^{-},[609-\text { glucosyl }]^{-}$, and $\left[609-\mathrm{C}_{12} \mathrm{H}_{20} \mathrm{O}_{9} \text { (rutinose) }\right]^{-}$, respectively.

The components of peak 15, 16, and 17 were temporarily identified as three flavonoids of kaempferol-3-O-glucoside, kaempferol-3-O-rutinoside (nicotiflorin), and quercetin-3O-rhamnoside [22, 23], respectively. The quasimolecular ions of peak 15 were at $m / z$ of $447.0945\left([\mathrm{M}-\mathrm{H}]^{-}\right)$, the ones of peak 16 were determined as $m / z$ of $593.1510\left([\mathrm{M}-\mathrm{H}]^{-}\right)$, and the ones of peak 17 were determined as $m / z$ as 447.0939 $\left([\mathrm{M}-\mathrm{H}]^{-}\right)$and $483.0705\left([\mathrm{M}+\mathrm{Cl}]^{-}\right)$. The MS/MS fragment ions of peak 15 were $\mathrm{m} / \mathrm{z}$ of 447.0940 due to $[\mathrm{M}-\mathrm{H}]^{-}$and 285.0404 due to $[\mathrm{M}-\mathrm{H} \text {-glucosyl }]^{-}$, the ones of peak 16 were $\mathrm{m} / z$ of 593.1525 and 285.0469 , and the ones of peak 17 were $\mathrm{m} / z$ of 447.0954 and 285.0420 .

The structures or possible structures of the components of peaks $1-8$ and peaks $10-20$ are shown in Figure 4 .
3.5. Wavelength Selection for Quantitative Analysis of 13 Components. It was found that all 13 components could be detected at $254 \mathrm{~nm}$, but the peak of 3,4-diCQA (peak 18) has not been completely separated from the nearby ones; organic acids had strong absorption near $324 \mathrm{~nm}$, but there was almost no absorption of $p$-hydroxyacetophenone (peak 8) at this wavelength. Therefore, $324 \mathrm{~nm}$ was selected as the detection wavelength for nine organic acids, and $254 \mathrm{~nm}$ was selected for other four components. The chromatograms of the mixed reference substances and sample are shown in Figure 5 .

3.6. Contents of 13 Components in 31 Batches of A. capillaris. As given in Table 5 and Figure 6, there were some differences in the contents of 13 components in different samples, which is basically consistent with the previous literature reports [12-14]; however, the content of CA seems to have a certain correlation with the other 12 components and the total of 13 components. Therefore, the bivariate correlation analysis method in SPSS 20 statistical software was used to analyze the correlation between the contents of CA and the contents of 12 other components and the total content of all 13 components in 31 batches of $A$. capillaris. As the results given in Table 6 , the contents of 10 components and the total content of 13 components were significantly correlated with the content of CA $(P<0.01$ or $P<0.05)$, except for the content of caffeic acid and 1,3-diCQA, which had poor correlation with the content of CA $(P>0.05)$. According to the data in Table 5, the average contents of caffeic acid and 1,3-diCQA in 31 batches of samples only account for $1.10 \%$ and $0.92 \%$ of the average contents of all 13 components, 


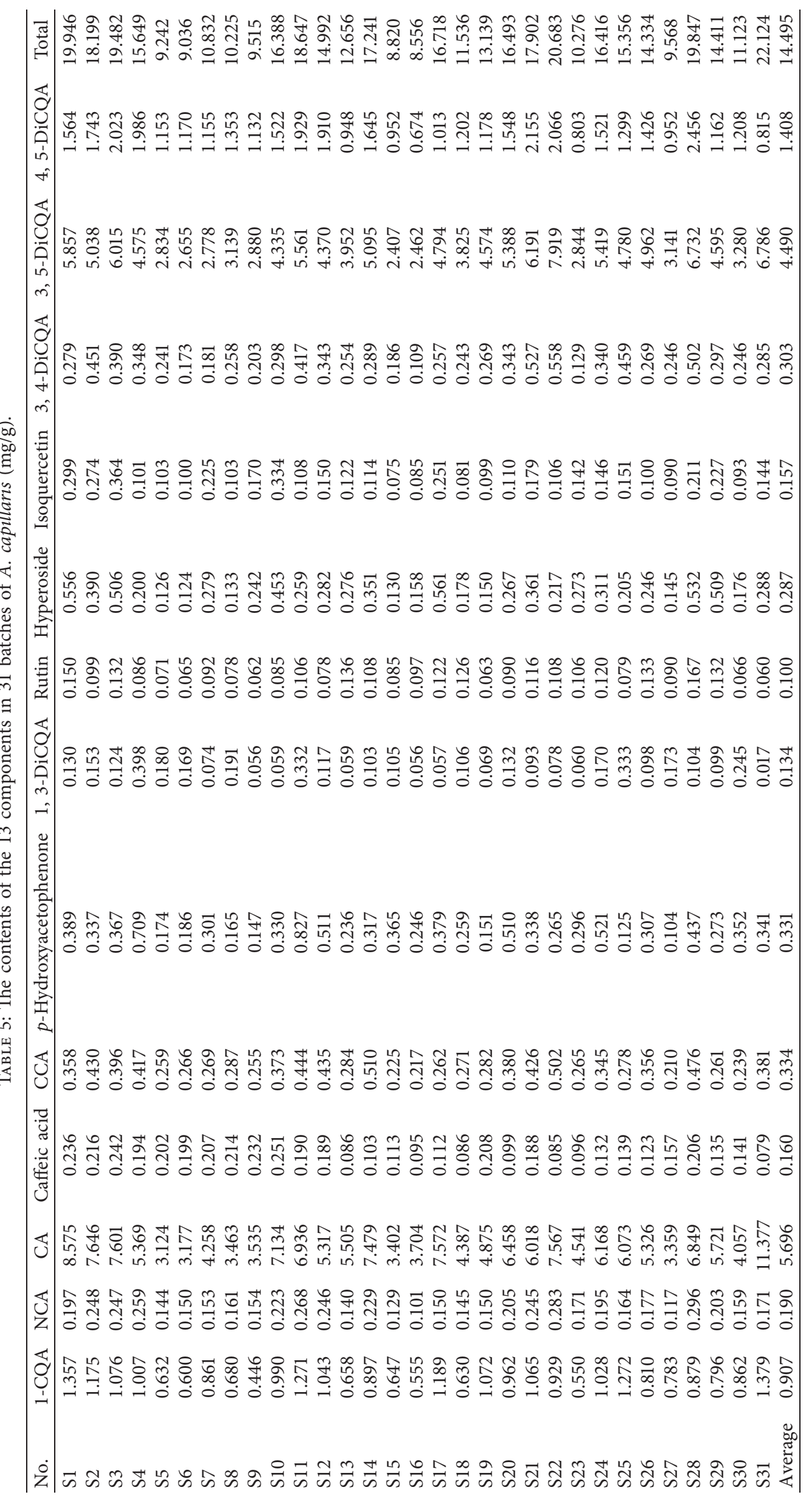




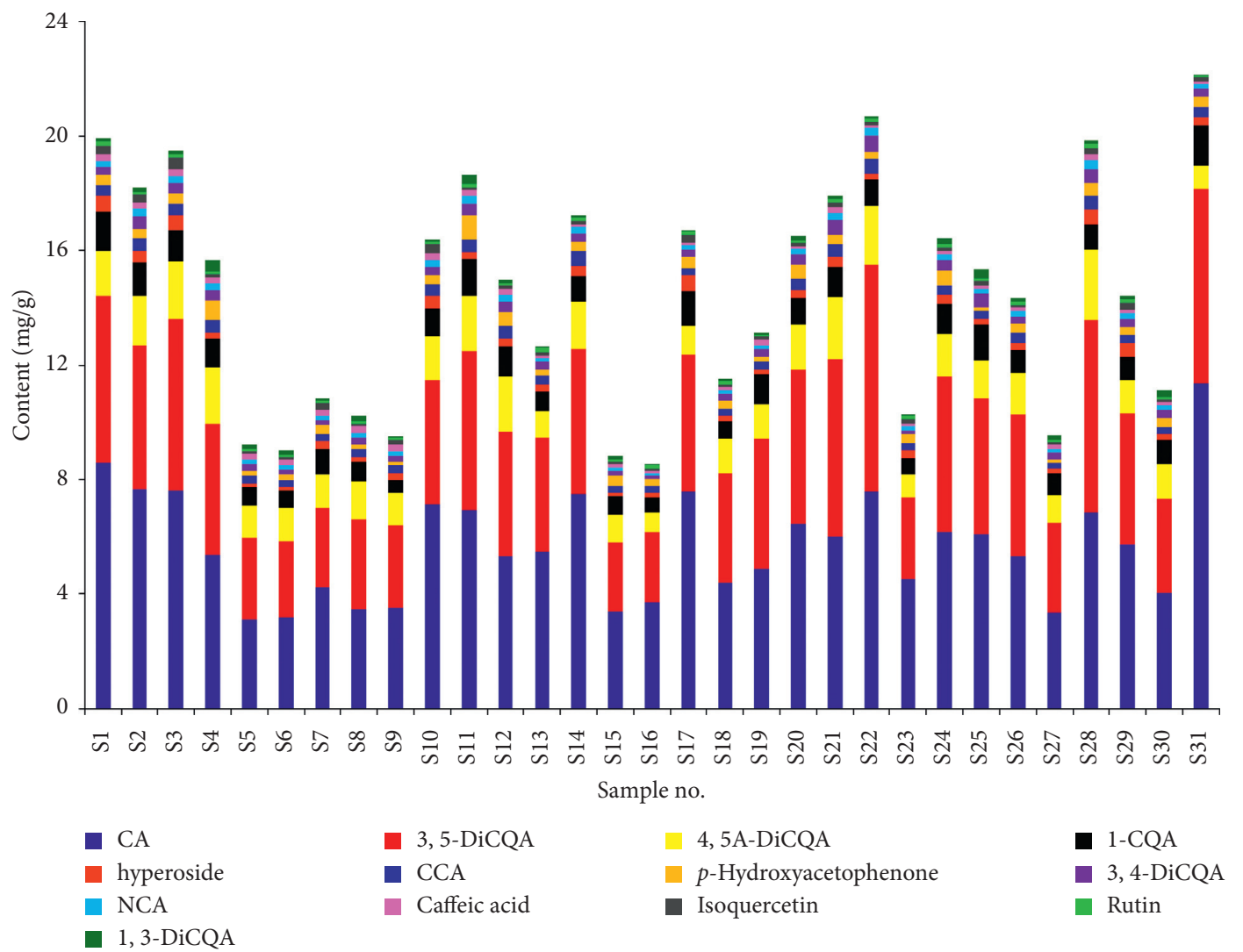

Figure 6: The bar graph of contents of 13 components in 31 batches of A. capillaris.

TABLE 6: Bivariate correlation analysis results of the correlation between the contents of CA and 12 other components and the total of all 13 components in 31 batches of A. capillaris.

\begin{tabular}{lcc}
\hline Component & $\begin{array}{c}\text { Correlation coefficient of } \\
\text { Spearson }\end{array}$ & $P$ \\
\hline 1-CQA & 0.748 & 0.0001 \\
NCA & 0.665 & 0.0001 \\
Caffeic acid & -0.340 & 0.856 \\
CCA & 0.698 & 0.0001 \\
p- & 0.529 & 0.002 \\
Hydroxyacetophenone & -0.160 & 0.390 \\
1,3-DiCQA & 0.444 & 0.012 \\
Rutin & 0.797 & 0.0001 \\
Hyperoside & 0.650 & 0.0001 \\
Isoquercetin & 0.696 & 0.0001 \\
3,4-diCQA & 0.861 & 0.0001 \\
3,5-diCQA & 0.498 & 0.005 \\
4,5-diCQA & 0.942 & 0.0001 \\
Total of 13 & & \\
\hline
\end{tabular}

respectively, so these two components can be ignored to a certain extent. According to the above analysis, the content of other components in different batches of A. capillaris is obviously related to the content of CA, that is to say, the content of CA largely reflects the quality of $A$. capillaris. Therefore, CA can be regard as the quality marker of A. capillaris. CA is the content determination component of A. capillaris in Chinese Pharmacopoeia. It is suggested that hospitals, pharmacies, and pharmaceutical manufacturers purchase multiple batches of $A$. capillaris and mix the high and low CA content batches before use according to the CA detection report provided by the supplier, so as to ensure clinical safety and effectiveness.

\section{Conclusion}

In this study, a new method was developed for the comprehensive QE of $A$. capillaris based on qualitative analysis of the HPLC fingerprint and UFLC-Q-TOF-MS/MS combined with quantitative analysis of multicomponents. The results showed that there were 20 common peaks in the HPLC fingerprints of $A$. capillaris. The similarities between the sample chromatograms and reference chromatogram were good. The components of the 20 common peaks were identified as ten organic acids, eight flavonoids, and two others. Among nine organic acids such as 1-CQA, NCA, CA, caffeic acid, CCA, 1,3-diCQA, 3,4-diCQA, 3,5-diCQA, and 4,5-diCQA, three flavonoids such as rutin, hyperoside, and isoquercetin, and one other $p$-hydroxyacetophenone, a total of 13 components were unambiguously identified by comparison with reference substances; one caffeoylquinic acid glucoside of $1-O-\left(4^{\prime}-O-\beta-D\right.$-glucosyl caffeoyl $)$ quinic acid or $1-O-\left(3^{\prime}-O-\beta\right.$-D-glucosyl caffeoyl $)$ quinic acid and one flavone di-C-glucoside of apigenin 6,8 -di- $C$ - $\beta$-D-glucoside were detected in A. capillaris for the first time. There were some differences in the contents of 13 components in 
different samples; chlorogenic acid could be regarded as the quality marker of $A$. capillaris. In summary, the method established in the present study can be used for the comprehensive QE of A. capillaris and can also provide reference for QE of other HMs.

\section{Data Availability}

The data used to support the findings of this study are included within the article.

\section{Conflicts of Interest}

The authors declare that they have no conflicts of interest.

\section{Authors' Contributions}

Rongrong Zhou and Zhihua Dou contributed equally to this work.

\section{Acknowledgments}

The authors would like to acknowledge KeyResearch and Development (Social Development) Fund Project of Jiangsu Province, China (BE2018674), Traditional Chinese Medicine Science and Technology Plan Project of Jiangsu Province, China (YB201836), and Nantong Basic Research Project, Nantong, Jiangsu Province, China (JCZ20168).

\section{References}

[1] X. Wu, H. Zhang, S. Fan et al., "Quality markers based on biological activity: A new strategy for the quality control of traditional Chinese medicine," Phytomedicine, vol. 44, pp. 103-108, 2018.

[2] X. Liu, W. Jiang, M. Su et al., "Quality evaluation of traditional Chinese medicines based on fingerprinting," Journal of Separation Science, vol. 43, no. 1, pp. 6-17, 2020.

[3] Y. J. Zhang, C. Wang, L. F. Yang, and G. X. Sun, “A strategy for qualitative and quantitative profiling of glycyrrhiza extract and discovery of potential markers by fingerprint-activity relationship modeling," Scientific Reports, vol. 9, no. 1, 2019.

[4] B. Yang, Y. Wang, L. L. Shan et al., "A Novel and practical chromatographic "fingerprint-ROC-SVM" strategy applied to quality analysis of traditional Chinese medicine Injections: using KuDieZi Injection as a case study," Molecules, vol. 22, no. 7, 2017.

[5] L. Jia, L. L. Fu, X. Y. Wang et al., "Systematic profiling of the multicomponents and authentication of Erzhi Pill by UHPLC/ Q-Orbitrap-MS oriented rapid polarity-switching data-dependent acquisition and selective monitoring of the chemical markers deduced from fingerprint analysis," Molecules, vol. 23, no. 12, 2018.

[6] T. Zhu, X. Liu, X. Wang et al., "Profiling and analysis of multiple compounds in rhubarb decoction after processing by wine steaming using UHPLC-Q-TOF-MS coupled with multiple statistical strategies," Journal of Separation Science, vol. 39, no. 15, pp. 3081-3090, 2016.

[7] Y. J. Cai, Q. Zheng, R. Sun et al., "Recent progress in the study of Artemisiae Scopariae Herba (Yin Chen), a promising medicinal herb for liver diseases," Biomedicine \& Pharmacotherapy, vol. 130, 2020.
[8] J.-M. Han, H.-G. Kim, M.-K. Choi et al., “Artemisia capillaris extract protects against bile duct ligation-induced liver fibrosis in rats," Experimental and Toxicologic Pathology, vol. 65, no. 6, pp. 837-844, 2013.

[9] C.-S. He, H.-Y. Yue, J. Xu et al., "Protective effects of capillary artemisia polysaccharide on oxidative injury to the liver in rats with obstructive jaundice," Experimental and Therapeutic Medicine, vol. 4, no. 4, pp. 645-648, 2012.

[10] F. Q. Guo, Y. Z. Liang, C. J. Xu, L. F. Huang, and X. N. Li, "Comparison of the volatile constituents of Artemisia capillaris from different locations by gas chromatography-mass spectrometry and projection method," Journal of Chromatography A, vol. 1054, no. 1-2, pp. 73-79, 2004.

[11] F. Yu, H. Qian, J. Zhang, J. Sun, and Z. Ma, "Simultaneous quantification of eight organic acid components in Artemisia capillaris Thunb (Yinchen) extract using high-performance liquid chromatography coupled with diode array detection and high-resolution mass spectrometry," Journal of Food and Drug Analysis, vol. 26, no. 2, pp. 788-795, 2018.

[12] F. Tian, Q. J. Ruan, Y. Zhang et al., "Quantitative analysis of six phenolic acids in Artemisia capillaris (Yinchen) by HPLC$\mathrm{DAD}$ and their transformation pathways in decoction preparation process," Journal of Analytical Methods in Chemistry, vol. 2020, 2020.

[13] X. J. Tan, Q. Li, X. H. Chen et al., "Simultaneous determination of 13 bioactive compounds in Herba Artemisiae Scopariae (Yin Chen) from different harvest seasons by HPLC-DAD," Journal of Pharmaceutical and Biomedical Analysis, vol. 47, no. 4-5, pp. 847-853, 2008.

[14] K. M. Park, Y. Li, B. Kim et al., "High-performance liquid chromatographic analysis for quantitation of marker compounds of Artemisia capillaris Thunb," Archives of Pharmacal Research, vol. 35, no. 12, pp. 2153-2162, 2012.

[15] Z. H. Dou, Y. Dai, Y. Z. Zhou et al., "Quality evaluation of rhubarb based on qualitative analysis of the HPLC fingerprint and UFLC-Q-TOF-MS/MS combined with quantitative analysis of eight anthraquinone glycosides by QAMS," Biomedical Chromatography, vol. 35, Article ID e5074, 2021.

[16] SFDA (State Food and Drug Administration of China), Technical Requirements for the Development of Fingerprints of TCM Injections, SFDA (State Food and Drug Administration of China), Beijing, China, 2000.

[17] China Medical Science Press, Pharmacopoeia of China, 2020. Part 4, China Medical Science Press, Beijing, China, 2020.

[18] H.-Y. Ma, Y. Sun, Y.-Z. Zhou, M. Hong, and Y.-H. Pei, “Two new constituents from Artemisia Capillaris Thunb," Molecules, vol. 13, no. 2, pp. 267-271, 2008.

[19] J.-Y. Zhang, Q. Zhang, N. Li, Z.-J. Wang, J.-Q. Lu, and Y.-J. Qiao, "Diagnostic fragment-ion-based and extension strategy coupled to DFIs intensity analysis for identification of chlorogenic acids isomers in Flos Lonicerae Japonicae by HPLC-ESI-MSn," Talanta, vol. 104, pp. 1-9, 2013.

[20] J. Cao, C. Yin, Y. Qin, Z. Cheng, and D. Chen, "Approach to the study of flavone di-C-glycosides by high performance liquid chromatography-tandem ion trap mass spectrometry and its application to characterization of flavonoid composition inViola yedoensis," Journal of Mass Spectrometry, vol. 49, no. 10, pp. 1010-1024, 2014.

[21] Y. Cao, T. Li, X. Xu et al., "Rapid chemical profiling of Artemisiae Scopariae Herba using reversed phase liquid chromatography-hydrophilic interaction liquid chromatography-predictive multiple reaction monitoring," China Journal of Chinese Materia Medica, vol. 44, no. 13, pp. 2667-2674, 2019. 
[22] W. Z. Ouyang, Z. P. Shang, W. J. Wang et al., "Rapid characterization of chemical constituents in capillary wormwood extract based on UHPLC-LTQ-Orbitrap," China Journal of Chinese Materia Medica, vol. 42, no. 3, pp. 523-530, 2017.

[23] Z. Fu, Y. Ling, Z. Li, M. Chen, Z. Sun, and C. Huang, "HPLCQ-TOF-MS/MS for analysis of major chemical constituents of Yinchen-Zhizi herb pair extract," Biomedical Chromatography, vol. 28, no. 4, pp. 475-485, 2014. 